Notre Dame Journal of Formal Logic

Volume 30, Number 3, Summer 1989

\title{
Logical Constants and the Glory of Truth-Conditional Semantics
}

\author{
WILLIAM G. LYCAN
}

\begin{abstract}
This paper endorses and defends M. J. Cresswell's view that the distinction drawn in linguistic semantics between strictly "logical" implication and merely lexical implication is bogus, and then explores the bad consequences that concession has for the Davidsonian semantic program. A pattern of semantic explanation made famous by Davidson's "The logical form of action sentences" is shown to be far less interesting than has been thought.
\end{abstract}

This paper commemorates Davidson's "The logical form of action sentences" [4]. In so doing it also celebrates the logical forms of action sentences. Those logical forms are still with us, since action sentences themselves are; but for linguistic semantics generally the magnificent promise of Davidson's classic article has never been fulfilled. My purpose here is to explore one reason that this is so.

1 According to (loosely) Davidsonian semantic theory, ${ }^{1}$ the core meaning of a sentence-its propositional or locutionary content as recorded in indirect discourse by a 'that'-clause - is that sentence's truth-condition. The sentence's truth-condition is determined by the meanings of the sentence's smallest meaningful parts together with their grammatical mode of composition, and it is best represented by a formula of some explicitly truth-defined logical system acting as a canonical idiom. Such a formula wears its own truth-condition on its sleeve, in that its truth-condition is computable on the basis of the usual Tarskian set of valuations for the atomic elements of that system plus a set of recursive rules that project the semantic values of a formula's elements through truth-functional and other syntactic compounding into a truth-condition for the formula as a whole.

So we have our original natural language sentence, endowed with its 
truth-condition as represented by its associated formula - call that explicitly truth-defined formula the sentence's semantic representation (SR). By means of its assigned SR, the target sentence's logical and semantical features are predicted, for logical relations are defined in the usual way over the formulas of the canonical idiom. In this way, too, logical anomalies are resolved and semantically puzzling features of the target sentences are explained, just as Russell imagined at the time he fashioned the Theory of Descriptions. Target sentences simply inherit their perceived semantical features, such as entailments, from the formal properties of their SR's.

To "do semantics" is to assign explicit truth-conditions to sentences. A semantic theorist investigates the semantics of a particular natural language by associating canonical SR's with the sentences of the language in such a way as to illuminate semantic structure consonantly with what is known of the sentence's syntactic structure. A semantics will have, or should have, testable consequences: it will predict ambiguities, synonymies, anomalies, logical implications, and the like. Capturing implications is a main goal, perhaps the main goal, of the enterprise.

Davidson did it (i.e., accomplished a capturing of implications) in the most dramatic way possible, for the case of action verbs and their most typical modifiers. Concentrating on the demodification inference (from 'Jones buttered the toast slowly, deliberately, in the bathroom, with a knife, at midnight' to 'Jones buttered the toast slowly, with a knife, at midnight', 'Jones buttered the toast deliberately, in the bathroom', 'Jones buttered the toast', and the like), Davidson hypothesized that the modified verbs are really existential quantifications over actions construed as concrete events, and that their modifiers are really conjoined predications of the corresponding bound event variables. Thus, the sentence 'Jones buttered the toast' would be represented as

(1) ( $\exists e$ )BUTTERING ( $e$, Jones, the toast)

('There occurred a buttering, by Jones, of the toast'), or more elaborately, picking up further obvious implications, as

$\left(1^{*}\right)$ ( $\exists e$ )(BUTTERING $(e) \&$ PROTAGONIST(Jones, $\left.e\right) \&$ VICTIM(the toast, $e)$ ).

Modifiers are just further conjoined predications, which explains why they may be stripped singly or in groups:

(2) ( $\exists e$ )(BUTTERING $(e$, Jones, the toast) \& SLOW(e) \& DELIBERATE $(e)$ $\&$ OCCURRED-IN $(e$, the bathroom) \& $(\exists y)(\operatorname{KNIFE}(y) \&$ DONE$\operatorname{WITH}(e, y)) \&$ OCCURRED-AT $(e$, midnight)).

So demodification is at bottom just ampersand-elimination, the trivial derivation of a conjunct from a conjunction. A puzzling and potentially troublesome felt implication is reduced to a clean, extensional, canonical, indeed classical form of inference. And so, captured.

But the notion of "capturing" involved here needs looking into.

2 The sentence 'Mort is incredibly lucky and either an idiot or a genius' is felt nonnegotiably to imply 'Mort is incredibly lucky'. The sentence 'Mort is a 
bachelor' is felt nonnegotiably to imply 'Mort is unmarried'. But there is a difference. The former implication is thought to hold in virtue of the target sentence's "logical structure", while the latter is only "lexical", a matter of individual word meaning, viz., of the meanings of 'bachelor' and of 'unmarried' (or perhaps 'married' as modified by 'un-'). I do not know how strongly this structuralvs-lexical difference is felt by ordinary English speakers, but it impresses itself on logicians and philosophers. Notice that any professional would casually grant that 'Mort is incredibly lucky and either an idiot or a genius' entails outright that 'Mort is incredibly lucky', while the felt implication of 'Mort is unmarried' by 'Mort is a bachelor' is not so easily described as entailment, even though it is apodeictic and noncancellable. Real entailment is a matter of syntactic/semantic structure, while implications that hold in virtue of simple word meaning are only halfheartedly felt to deserve the title of "entailment", even when they seem equally apodeictic and noncancellable.

In a linguistic theory built along Davidsonian lines many felt implications are captured or codified structurally, but many others, lexical ones, are left over. The theory must deal with them in some different way. The most popular way is the introduction of "meaning postulates", or, as Harman [6] once called them, "nonlogical axioms":

\begin{tabular}{|c|}
\multicolumn{1}{|c|}{ Meaning Postulates } \\
$(x)(\operatorname{BACHELOR}(x) \rightarrow \operatorname{ADULT}(x))$ \\
$(x)(\operatorname{BACHELOR}(x) \rightarrow \sim \operatorname{MARRIED}(x))$ \\
$(x)(\operatorname{BACHELOR}(x) \rightarrow \operatorname{MALE}(x))$.
\end{tabular}

Meaning postulates can themselves employ forthrightly logical operators such as negation.

Now, notoriously, Quine has taken a dim view of "meaning postulates" as originally introduced by Carnap. Simply by writing the heading "Meaning Postulates" above the list of generalizations that have been claimed to be analytic, one does nothing to explicate analyticity or synonymy. Nor, in a linguistic theory, does one go anywhere toward explaining a felt implication by writing "Meaning Postulate" above a representation of that implication. One may thereby record one's impression that two or more concepts are connected in a logical/ linguistic manner rather than an empirical one, but such impressions are of no theoretical significance unless they are themselves explained.

Quine of course denies that there is any theoretically substantive difference between generalizations that hold in virtue of word meaning and those that are simply empirical correlations in the world. Thus he seeks no substitute for "meaning postulates", but would advise simply scrapping the idea of "lexical semantics" even if he were to tolerate linguistic semantics more generally.

I am sympathetic to Quine's unsympathetic view and have elsewhere defended it myself [8]. But I am now afraid it will not do as it stands, for the distinction between mere "meaning postulates" and bonafide logical truths proves to be deeply suspect.

3 A "logical truth" is supposed to be a sentence or formula that is true under any admissible reinterpretation of its nonlogical terms; nonlogical terms, that 
is, as opposed to logical constants. Logical constants are thus presupposed to differ in kind from ordinary morphemes. But this presupposition is not at all obviously correct. Various authors have encountered difficulties in trying to delineate the class of logical constants in any principled way (see Peacocke [12], McCarthy [9]). Even worse, as has not been generally noticed, mere lexical implications seem to shade off into clearly logical entailments by fairly smooth degrees. Consider:

$x$ is a bachelor $\rightarrow x$ is unmarried

$x$ has a daughter $\rightarrow x$ is a parent

$x$ is red $\rightarrow x$ is colored

$x$ is a closed curve in a plane $\rightarrow x$ has an area

$x$ is a hexagon $\rightarrow x$ is a regular polygon

$x V^{\prime}$ 'ed $A d v$ ly $\rightarrow x V^{\prime}$ 'ed

$x$ has been un $V^{\prime}$ 'ed $\rightarrow x$ was $V^{\prime}$ 'ed

$x$ is very $A d j \rightarrow x$ is $A d j$

$x$ is now $F \rightarrow$ It will be the case that $x$ was $F$

$x$ knows that $P \rightarrow P$

Necessarily $P \rightarrow P$

$x$ is greater than $y$ and $y$ is greater than $z \rightarrow y$ is greater than $z$

Most $F$ 's are $G \rightarrow$ Many $F$ 's are $G$; Many $F$ 's are $G \rightarrow$ Some $F$ 's are $G$;

Some $F$ 's are $G \rightarrow$ At least one $F$ is $G$

There are $n F$ 's $\rightarrow$ There are at least $n-m F$ 's (for integers $n$ and $m, 1 \leqq$ $m \leqq n-1)$

$x$ is identical with $y \rightarrow y$ is identical with $x ; x$ is identical with $y$ and $y$ is identical with $z \rightarrow x$ is identical with $z$

All $F$ 's are $G \rightarrow$ Some $F$ 's are $G$

There is an $F$ that $V$ 's every $G \rightarrow$ Every $G$ is $V^{\prime}$ 'd by some $F$

$R$, and either $R$ and $Q$ or $R$ and not $P \rightarrow$ If $P$ then $Q$

$P$ and $Q \rightarrow P$.

The foregoing examples are listed roughly in increasing order of "logicalness". There is plenty of room for dispute about exact placement of the examples. (Is 'greater than' really more structural or logical, i.e., less purely lexical, than 'necessarily'?) And there would be even more dispute about other examples, e.g., where do various odd sorts of modifiers fit in? But the general moral is clear: in no such good healthy list of examples can any great obtrusive break be seen, between the merely lexical and the genuinely "structural". Logicalness as opposed to lexicalness seems to be a matter of degree or at best of grade.

The point was made years ago by Cresswell, specifically in regard to Davidson's demodification project but also addressing some of Davidson's nonclassical, antiextensionalist opponents in the ensuing literature:

All these authors [Davidson [4], Montague [10], Parsons [11], Lewis [7], and Clark [1]] seem to have at the back of their minds that (a) it is possible, and (b) it is desirable, to make explicit some or all of the true entailments between English sentences. I am pretty sure that it is not possible to do it for all true entailments and I see little theoretical interest in doing it only for some. The sentence this is red entails this is coloured because the meaning 
of these sentences in English is such that in any possible world if it is true to say this is red it is also true to say this is coloured. Whether an English speaker has come to learn the meaning of red by building it up from meaning blocks which include the meaning of coloured is of no concern to the logician. ([3], p. 470)

In establishing the format of his own great semantical work, Logics and Languages [2], Cresswell declined to distinguish any set of "logical" words of English from ordinary morphemes. (Though somewhat incongruously he did treat a word of non- or pre-English as purely logical: the intensional abstraction operator $\lambda .{ }^{2}$ On Cresswell's theory, $\lambda$ mediates countless felt implications, but it is grammatically deleted from every sentence everywhere and, oddly, has no English lexical realization at all-a truly modest hero. On this understanding, Cresswell should be read not as challenging the "logical"/"nonlogical" distinction, but simply as maintaining that in fact the English lexicon happens to contain only nonlogical terms, English's one logical constant being systematically suppressed by syntax.)

The Cresswell passage quoted above is striking in that it opposes a realist view of the logical/lexical distinction with only a psychological claim about learning. Evidently Cresswell sees the prevailing conception of a genuinely logical entailment as requiring something to do with a psychological process of building up concepts. But what? One might suppose that an entailment obtains when the entailing proposition requires the entailed proposition in the psychological order of learning. But that criterion would let in any lexical implication that in fact satisfies the psychological condition of de facto learning-theoretic priority; it has nothing to do with logic. A stronger criterion would be one according to which entailment requires psychologically real lexical decomposition, synchronic rather than developmental; but that criterion too could well let in implications arising from purely lexical decompositions, e.g., as of ' $x$ kill $y$ ' into ' $x$ cause: $y$ die'. The most obviously next stronger requirement is that genuine entailments should be explicated in terms of psychologically real lexical decomposition plus genuinely logical constants; e.g., 'This is red' entails 'This is colored' only if psychologically the English 'red' decomposes into (hence one can learn 'red' only by first learning) both 'colored' and the ampersand, and in effect 'red' abbreviates the concatenation of 'colored', the ampersand, and some third concept that somehow determines the determinable-but that criterion is (at best) unlikely to be satisfied, and is circular to boot.

I suspect that what Cresswell meant to attribute to his opponents is the second of the foregoing three criteria, the idea being that if an implication really is authorized by a psychologically real lexical decomposition then that implication is as genuine a case of entailment as anyone might wish, and other "implications" that do not meet the psychological standard simply do not qualify. On this exegesis, Cresswell reacted with a logician's quite proper instinct to distinguish a logical concept from all matters of psychology. Proponents of what the Generative Semanticists of the late 1960's called "Natural Logic" would balk at this, and we should all agree that a real psychological distinction is a genuine distinction and, moreover, one to be scrupulously respected by linguistic semantics, but logic does not automatically include all of linguistic semantics, and I would agree with Cresswell (on the present interpretation) that "psychological 
entailment" of this sort is not eo ipso logical entailment in anything like the sense usually attached to that term. ${ }^{3}$

But there is a halfway house. We can read "The logical form of action sentences" anachronistically as an attempt to save the "logical"/"lexical" distinction by showing that intermediate or dubious cases of logical implication reduce to bonafide entailment when real logical form is ultimately revealed, "real" logical form involving only something like the traditional logical operators. And a psychological-reality claim for that revelation would strongly support a special logical status for the relevant class of fundamental operators. Thus there would be a middle ground between the obviously lexical and the obviously logical locutions of English: those which are not logical on the surface but which semantically decompose into fundamental logical operators and nothing but. That middle ground is Davidson's strongest hope.

4 If logicalness as opposed to lexicalness is a matter of degree, what more basic magnitude controls that degree? Generality of subject matter is the obvious candidate. 'Bachelor' applies to very few individuals, cosmically speaking, while 'and' is useful coinage anywhere; 'know' is restricted to sapient subjects, while 'greater than' applies to anything that comes in degrees but to nothing else. I think Peacocke [12] had the right idea in suggesting that "topic-neutrality" is intuitively the key desideratum: logic is supposed to be abstract and utterly catholic, in no way attached to any particular topic of conversation. Does that guiding idea not mark off some locutions from the rest?

Peacocke and then McCarthy [9] maintained that it does, and both authors have constructed formal devices intended to implement the idea of topicneutrality. But intuitively, once one starts thinking in terms of a continuum of cases, topic-neutrality itself in effect comes in degrees. The locutions featured in my list of examples differ in the degree to which they constrain the ontology of the expressions to which they apply, but only for the case of truth-functional connectives is it strictly right to say that there is no constraint whatever. Adverbial demodification is about human or nonhuman agents. Set theory is at least about sets. Quantification theory is at least about a domain of individuals and either sets or properties defined on that domain. One might for this reason insist that only the truth-functional connectives are genuinely logical constants, and not even the quantifiers qualify; I would accept that as a well-motivated stipulation, but only as a stipulation. (N.B.: agreement with Cresswell's continuum thesis does not rule out the possibility of defining sharp distinctions near the upper end of the continuum of "logicalness". For example, one might accept a cleaned-up version of Peacocke's distinction having to do with de re a priori knowledge as a genuine distinction, and even as providing for some purposes a good meaning for 'logical constant', without committing oneself to the claim that, as a matter of fact, all and only expressions meeting the Peacocke condition are true logical constants.)

There is a possible competing candidate for the magnitude underlying degrees of logicalness, suggested by Quine [13]. At one point in his chapter on logical truth he suggests tying the "logical"/"lexical" distinction to the grammatical notion of a particle: logical constants are particles rather than members of what we might call the lexicon at large. Particles are distinguished from ordi- 
nary lexical items in virtue of their falling into small grammatical categories whose membership is fixed. By contrast, lexical categories are indefinite in their membership, typically infinite owing to the recursive compounding of complex expressions. ${ }^{4}$ Now, this Quinean view could naturally survive the rejection of an absolute "logical"/"lexical" distinction, for the size of a grammatical category is a matter of degree as well. Thus it might be suggested that "logicalness" is at bottom a matter of that size, i.e., of how few other morphemes there are of the same grammatical type.

I think it is clear that that suggestion will not do. For the actual size of a grammatical category's membership is a highly contingent fact about a given natural language, and it is not a priori related to the logical status of any of the expressions that are the category's members. Moreover, if it is iterative compounding that makes the categories of, say, singular terms and common nouns indefinitely large and open-ended, the same applies to the category of binary truth-functional connectives, for English contains nonfinitely many well-formed complex connectives, even though only a few of them are ever actually used and all but sixteen of them are logically equivalent to simpler ones. Thus we had better stick to degrees of generality or topic-neutrality after all.

5 If I am right in agreeing with Cresswell that the "logical"/"lexical" distinction is one of degree rather than one of kind, that in turn impugns the distinction between the official truth-rules that define logical operators (the operators' recursion clauses in a Tarskian truth definition for the containing language) and the measly, pathetic "meaning postulates" scribbled down ad hoc to "explain" purely lexical implications. What then becomes of Quinean skepticism about the latter?

Quine's view is that while the recursion clauses in a logical theory may be said to play an explanatory or at least substantive systematizing role in regimenting the sentences of a natural language and exhibiting patterns of valid inference, the heading 'meaning postulates' is flatus vocis, an empty and pointless gesture of no explanatory value whatever. But once we see logicalness as a matter of degree and look back at typical meaning postulates in that spirit, we find that meaning postulates are not entirely empty. Even the most vapid of them affords at least a bit of generality: $(x)(\operatorname{BACHELOR}(x) \rightarrow \sim \operatorname{MARRIED}(x))$ subsumes 'If Reg is a bachelor Reg is unmarried', 'If Irv is a bachelor Irv is unmarried', and 'If Ronald Reagan is a bachelor Ronald Reagan is unmarried' as lexical truths.

I would contend that such humble subsumptions are authentically explanatory, though the explanations provided are very shallow. Why does 'Ronald Reagan is a bachelor' entail 'Ronald Reagan is unmarried'? Because the meanings of 'bachelor' and 'unmarried' are such that " $N$ is a bachelor" implies " $N$ is unmarried" no matter what singular term is uniformly substituted for ' $N$ '. Why does 'Ronald Reagan is unmarried and he believes in kirlian photography' entail 'Ronald Reagan is unmarried"? Because the meaning of 'and' is such that " $S$ and $T$ " implies " $S$ " no matter what sentence is uniformly substituted for ' $S$ ' and ' $T$ '. There is no difference in kind between these two explanatory statements, but only a difference in degree of generality. 
Quine thinks of meaning postulates as offering only "dormitive virtue" explanations. So do I. But against Quine and Voltaire I maintain that "dormitive virtue" explanations are genuine explanations, even though slight ones. When Dr. Pangloss proposes that laudanum puts people to sleep because it has a dormitive virtue, he succeeds in ruling out some alternatives, even though they are not very interesting or appealing alternatives. For laudanum to have a dormitive virtue is for any quantity of it to have or contain a power. People given laudanum have gone to sleep, not by chance, not by magic, and not even because a powerful god capriciously but uniformly chooses to put people to sleep when he notices that they have been given laudanum; they have gone to sleep because laudanum has something in it that itself puts people to sleep. Again, that is not an exciting or even very illuminating hypothesis, since few philosophers would have thought of doubting it, but it does rule out some conceivable alternatives and so is nonempty. ${ }^{5}$ To explain is (at least) to rule out alternative generalizations, and to explain powerfully or illuminatingly is to rule out more and/or whole ranges of alternatives. The number of alternatives excluded and the number of ranges of alternatives excluded are matters of degree. ${ }^{6}$ Thus meaning postulates and "dormitive virtue" explanations generally do genuinely explain; their shame is only that they do not explain very much. The Quine-Voltaire objection, as originally stated, evaporates.

6 But have we not done away with the principal motive, or at least Davidson's motive in "The logical form of action sentences," for doing truth-theoretic semantics in the first place? That motive was to "capture" felt implications by assimilating them to "logical" implications-more boldly, by showing them to be logical entailments, really, beneath the surface. Recall the wonderful rush of understanding produced by Davidson's analysis of action sentences (whether or not one agreed with its details, as I am sure no one did): the rendering of adverbial modifiers as logical conjuncts was a gorgeous paradigm case of reducing the unfamiliar (and mysterious) to the familiar by means of a clever hypothesis. Only the maddest logical conservative would think that Davidson's extensionalist treatment would work for all of a natural language - that the first-order predicate calculus alone is the underlying logic of English in particular. But it was not farfetched to imagine that first-order logic supplemented by some further logical operators would do. And if such an underlying logic were recoverable, then Davidson's great pattern of semantic explanation could have been extrapolated to all of human language.

But the Davidsonian project still presupposes the distinctness and the distinctiveness of a smallish identifiable class of genuinely logical operators. That presupposition of "genuinely logical" operators is just what I have been giving up. Moreover, it seems we must lose an important point of testability in a semantic theory: if there is no principled difference between a rule of logic and a mere "meaning postulate", and if any felt implication can be "captured" on the spot by the proclaiming of a meaning postulate, then felt implications are not the remorseless, implacable proving ground for semantics that they were intended to be.

Finally, our Cresswellian concession lets much of the air out of paraphrase 
arguments in semantics. It is usually supposed that if two sentences are synonymous or mutually paraphrase each other, then they probably (but not necessarily) share the same or logically equivalent logical forms. This assumption guides theorizing in a useful way. But, again, if there is no principled difference between a rule of logic and a mere "meaning postulate" our two sentences may be related only by meaning postulates and have nothing at all in common that we would think of as formal. The idea of a "logical form" itself threatens to attenuate. (I say only "threatens", because it is kept from harm in Cresswell [2] owing to that work's preservation of the non-English intensional abstraction operator $\lambda$ as a truly logical constant.)

7 What is left? We have abandoned the idea of a small subclass of genuinely logical words within the English lexicon. There are only degrees of "logicalness", which I have proposed to understand as degrees of generality of application. There may be one or more significant psychological distinctions to be made along the continuum, but that cannot be known a priori and in any case is of no concern to the logician.

One can of course join Cresswell in positing a non-English logical operator and tracing all implication in English to that operator. That is an attractive option, and preserves the idea of English as a formal language. Failing that, a semanticist is left, in regard to the capturing of implications, with only the aim of explaining implications in as "logical" terms as possible, other things being equal. That is, the methodological instruction to explain implications logically and eschew "meaning postulates" gives way to a preference for the more logical, which on my view is just a special case of one's common theoretical preference for the more general and the more unified. That preference is no biting empirical desideratum, but is weak and very easily overridden.

Perhaps Harman foresaw that such a weakening would be necessary when he expressed one of his early principles of theory preference as: "[M]inimize [nonlogical] axioms" ([6], p. 42). A straight-down-the-middle Quinean on the issue of meaning postulates would have said no such thing, but would simply have reminded the reader that so-called "nonlogical axioms" are bunk. On the other hand, the idea of minimizing nonlogical axioms presupposes that there are identifiable nonlogical axioms to be minimized, and unlike Harman we have abandoned that presupposition as well. There are neither logical operators with their mighty recursion clauses nor nonlogical axioms written in a special box, but only operators with their truth rules differing only in their generality of application. The relevant methodological instruction is simply to prefer the more general operators where one can, and as we have seen, that instruction has little authority.

Thus Clark's [1] response to Davidson and his competing treatment of adverbial modification are considerably more attractive than some of his critics have granted. Clark botanized adverbial modifiers into a number of different categories and wrote a separate truth rule for each. Harman [6] and others saw those truth rules as nonlogical axioms, and faulted Clark for proliferating such things. But though they are far from being "purely" logical, Clark's rules have a fair degree of generality, and are not just vapid bottom-level "meaning postulates" either; they are somewhere in the middle, and that is quite all right. 
These findings have in no way dimmed my admiration for "The logical form of action sentences". But they have taken away my hope that Davidson's achievement can be replicated throughout all fragments of natural languages everywhere-unless the psychological "middle ground" hypothesis formulated at the end of Section 3 should turn out to be true, as is unlikely.

\title{
NOTES
}

1. Whose roots are in Plato, Leibniz, Frege, Russell, Wittgenstein's Tractatus, and the early writings of Hintikka and Montague-Davidson's own distinctive contributions having been: (i) to implement all those theorists' notions of correspondence and/or picturing by way of Tarski's theory of truth, and (ii) by (i) to make semantics into a testable enterprise.

2. "Unlike any of the symbols we have met so far, $\lambda$ is a logical constant with a fixed interpretation" (p. 84). The principles of $\lambda$-conversion are "the only principles which are independent of a particular value assignment" ([2], p. 470).

3. I am less sympathetic to Cresswell's succeeding example and argument, to wit:

\begin{abstract}
If we try to mark off a class of entailments which depend only on the 'logical words' of English we are faced with the invidious task of deciding what these are. E.g., is 'if' one of them? If it is and if, as seems likely, 'if' in English is not truthfunctional then our logic will have to be some, as yet undiscovered, highly intensional logic. It is of no use to reply here that ' $i f$ ' when understood as material implication is a logical word of English unless we can sort out those sentences in which it is being so understood. . . .
\end{abstract}

I do not see the force of this. Why does non-truth-functionality automatically make an operator a problem case, unless one simply means to stipulate (as Cresswell expressly does not) that only truth-functional operators count as logical? Nor does the undiscoveredness of the intensional logic militate against 'if"s being genuinely logical, unless having been discovered is stipulatively and rashly required.

4. Harman [5] appeals to this criterion in arguing that modal operators are not logical constants.

5. For a similar defense of dormitive virtues against Voltaire, see Sober [14].

6. Though here (as ubiquitously in philosophy) the idea of degree along with that of proportion is officially stymied by the nondenumerability of the containing population, under a suitably fine-grained mode of individuation.

\section{REFERENCES}

[1] Clark, R., "Concerning the logic of predicate modifiers," Noûs, vol. 4 (1970), pp. 311-335.

[2] Cresswell, M. J., Logics and Languages, Methuen, London, 1973.

[3] Cresswell, M. J., “Adverbs and events,” Synthese, vol. 28 (1974), pp. 455-481.

[4] Davidson, D., "The logical form of action sentences," in The Logic of Decision and Action, edited by N. Rescher, University of Pittsburgh Press, Pittsburgh, 1967. 
[5] Harman, G., “Is modal logic logic?” Philosophia, vol. 2 (1972), pp. 75-84.

[6] Harman, G., "Logical form," Foundations of Language, vol. 9 (1972), pp. 38-65.

[7] Lewis, D., "General semantics," in Semantics of Natural Language, edited by D. Davidson and G. Harman, D. Reidel, Dordrecht, 1972.

[8] Lycan, W. G., Logical Form in Natural Language, Bradford Books/MIT Press, Cambridge, MA, 1984.

[9] McCarthy, T., "The idea of a logical constant," Journal of Philosophy, vol. 78 (1981), pp. 499-523.

[10] Montague, R., "Universal grammar," Theoria, vol. 36 (1970), pp. 373-398.

[11] Parsons, T., "Some problems concerning the logic of grammatical modifiers," in Semantics of Natural Language, edited by D. Davidson and G. Harman, D. Reidel, Dordrecht, 1972.

[12] Peacocke, C., "What is a logical constant?," Journal of Philosophy, vol. 73 (1976), pp. 221-240.

[13] Quine, W. V., Philosophy of Logic, Second edition, Harvard University Press, Cambridge, MA, 1986.

[14] Sober, E., "Dispositions and subjunctive conditionals, or, why dormitive virtues are no laughing matter," Philosophical Review, vol. 91 (1982), pp. 591-596.

Department of Philosophy University of North Carolina

Chapel Hill, North Carolina 27599-3125
Center for Philosophy of Science

817 Cathedral of Learning

University of Pittsburgh

Pittsburgh, Pennsylvania 15260 